\title{
不含烷基金属的丁二烯順式-1,4 聚合的可溶性催化剂
}

\author{
謝洪泉李平生金鷹泰
}

(中国科学院应用化学研究所)

Mazzei 等[1] 曾报导了某种不含烷 基金属的催 化剂聚合丁二烯的动力学，但末指出该催化剂的组 分。不久前 Marconi [2] 曾发表了用无机 化合物 卤氫化铝的衍生物与卤化鈦组成的非均相催化剂可 使丁二烯进行顺式-1，4聚合。我们发现 $\mathrm{AlHCl}_{2}$ 的 乙醚络合物与 $\mathrm{CoCl}_{2}$ 的吡啶络合物 及 $\mathrm{AlBr}_{3}$ 组成 的三元可溶性催化 剂可以作为丁二烯的顺式 $-1,4$ 聚合催化剂*, 所得聚合物的顺式-1, 4 结构很高, 达 $95 \%$ 以上，分子量可控制在 $20-30$ 万。

聚合操作均在纯氮保护下于干燥仪器中进行, 详细步骤见作者以前的报导 [3]。 $\mathrm{AlHCl}_{2}$ 的乙醚络 合物系按 Wiberg 及 Schmidt 的方法 ${ }^{[4]}$ 制得, 分析 结果为 $\mathrm{Al}: \mathrm{Cl}_{2}$ : 活性氫 $=1: 2: 0.75 ;$ 无水三澳化铝 按Nicholson 等的方法 ${ }^{[5]}$ 合成; 氯化鋿的吡啶络合 物按 Katzin 等的方法 ${ }^{[6]}$ 制得。聚合条件为单体浓

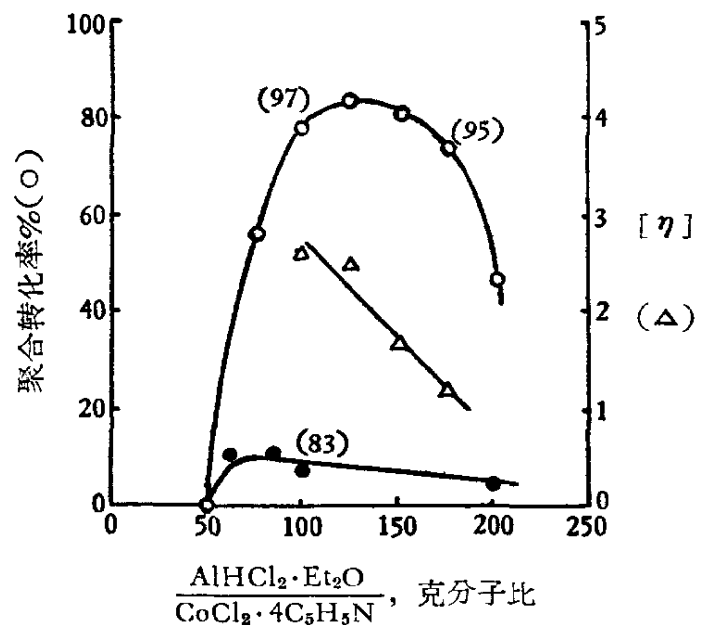

图 $1 \mathrm{AlHCl}_{2} \cdot \mathrm{Et}_{2} \mathrm{O}$ 与 $\mathrm{CoCl}_{2} \cdot 4 \mathrm{C}_{5} \mathrm{H}_{5} \mathrm{~N}$ 的克分 子比对聚合转化率及产物分子量的影响 $\left(30^{\circ} \mathrm{C}\right.$, $\mathrm{Co} / \mathrm{M}=50 \mathrm{ppm} ; 0, \Delta-\mathrm{AlBr}_{3} / \mathrm{AlHCl}_{2} \cdot \mathrm{Et}_{2} \mathrm{O}$ $=0.5,3$ 小时; - $-\mathrm{AlBr}_{8} / \mathrm{AlHCl}_{2} \cdot \mathrm{Et}_{2} \mathrm{O}$ $=0,17$ 小时)
度100 克/升,水分控制在 25ppm 左右, 聚合 3 小时。 $\mathrm{AlHCl}_{2}$ 本身不稳定, 且不溶解; 而 $\mathrm{AlHCl}_{2}$. $\mathrm{Et}_{2} \mathrm{O}$ 很稳定, 扭溶于苯中。单独 $\mathrm{AlHCl}_{2} \cdot \mathrm{Et}_{2} \mathrm{O}$ 与 $\mathrm{CoCl}_{2} \cdot 4 \mathrm{C}_{5} \mathrm{H}_{5} \mathrm{~N}$ 组成的可溶性催化剂活性不高, 在 $30^{\circ} \mathrm{C}$ 聚合 17 小时的转化率不到 $10 \%$, 活性 $\mathrm{Al} / \mathrm{Co}$ 比 (指 $\mathrm{AlHCl}_{2} \cdot \mathrm{Et}_{2} \mathrm{O} / \mathrm{CoCl}_{2} \cdot 4 \mathrm{C}_{5} \mathrm{H}_{5} \mathrm{~N}$ 的克分子比) 在 50-200 之间 (图 1)，顺式-1，4含量仅占 $83 \%$ 左 右, 推测这可能是乙䤓的量较多 (与 $\mathrm{AlHCl}_{2}$ 之比 为 $1: 1$ ), 抑制了 $\mathrm{AlHCl}_{2}$ 与 $\mathrm{CoCl}_{2}$ 生成活性中心。 根据以前研究的结果 ${ }^{[7]}, \mathrm{Et}_{2} \mathrm{O} / \mathrm{Al}$ 比 $>0.5$ 时, 聚 合被抑制。因此考虑加入亲电子试剂 $\mathrm{AlBr}_{3}$, 企图 使部分 $\mathrm{Et}_{2} \mathrm{O}$ 与 $\mathrm{AlBr}_{3}$ 络合 (一般络 合比为 $1: 1$ ), 其时释出的 $\mathrm{AlHCl}_{2}$ 可能与 $\mathrm{CoCl}_{2} \cdot 4 \mathrm{C}_{5} \mathrm{H}_{5} \mathrm{~N}$ 生成活

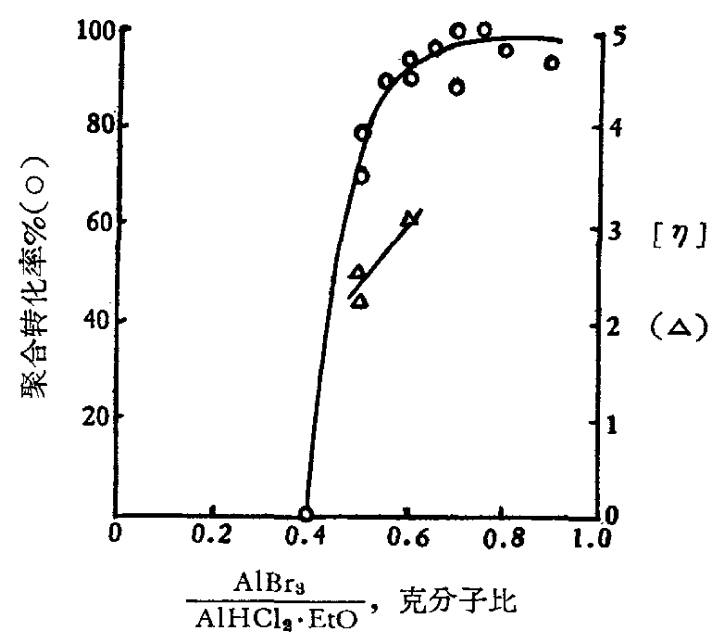

图 $2 \mathrm{AlBr}_{3}$ 与 $\mathrm{AlHCl}_{2} \cdot \mathrm{Et}_{2} \mathrm{O}$ 的克分子比对聚 合转化率及产物分子量的影响 $(\mathrm{Co} / \mathrm{M}=50 \mathrm{ppm}$, $\mathrm{AlHCl}_{2} \cdot \mathrm{Et}_{2} \mathrm{O} / \mathrm{CoCl}_{2} \cdot 4 \mathrm{C}_{5} \mathrm{H}_{5} \mathrm{~N}=100$ )

* 在我们工作的同时见到 C. A., 60, 10905 (1964) 中载有 H. Winter 及 $\mathrm{H}$. Weber 的专利摘要, 与 我们的发现有些类似之处, 但有相当的差別。 
性中心。结果发现, 加入 $\mathrm{AlBr}_{3}$ 占 $\mathrm{AlHCl}_{2} \cdot \mathrm{Et}_{2} \mathrm{O}$ 的 2/5 以上时，催化剂的聚合活性大增 (图 2)，3 小时的转化率可达 $70 \%$ 以上, 聚合 物的 顺式-1,4 结构也大增，一般可达 $96 \%$ 左右。活性随 $\mathrm{AlBr}_{3}$ / $\mathrm{AlHCl}_{2} \cdot \mathrm{Et}_{2} \mathrm{O}$ 的克分子比增加而增加。但如 $\mathrm{AlBr}_{3} /$ $\mathrm{AlHCl}_{2} \cdot \mathrm{Et}_{2} \mathrm{O}$ 比超过 0.6 时, 则聚合物中生成大量 凝胶。这可能是由于 $\mathrm{AlBr}_{3}$ 本身能作为阳离子聚合 催化剂, 过量会引起聚合物链发生交联。

固定 $\mathrm{AlBr}_{3} / \mathrm{AlHCl}_{2} \cdot \mathrm{Et}_{2} \mathrm{O}$ 比为 0.5 及 $\mathrm{CoCl}_{2}$. $4 \mathrm{C}_{5} \mathrm{H}_{5} \mathrm{~N}$ 用量为单体的 $50 \mathrm{ppm}$, 变化 $\mathrm{AlHCl}_{2} \cdot \mathrm{Et}_{2} \mathrm{O} /$ $\mathrm{CoCl}_{2} \cdot 4 \mathrm{C}_{5} \mathrm{H}_{5} \mathrm{~N}$ 的克分子比, 结果列于图 1 中, 可 见 $\mathrm{Al} / \mathrm{Co}$ 比在 50 以下，活性很小。 $\mathrm{Al} / \mathrm{Co}$ 比在 75-175 之间, 活性最大, 超过 175 则活性下降。 $\mathrm{Al} / \mathrm{Co}$ 比范围较 $\mathrm{Et}_{2} \mathrm{AlCl}-\mathrm{CoCl}_{2} \cdot 4 \mathrm{C}_{5} \mathrm{H}_{5} \mathrm{~N}$ 催化系 统 ${ }^{[8]}$ 的为狭。推测这是由于 $\mathrm{AlHCl}_{2} \cdot \mathrm{Et}_{2} \mathrm{O}$ 量少时 为聚合系统中的极性杂质所消徰, 以致不足以与 $\mathrm{CoCl}_{2} \cdot 4 \mathrm{C}_{5} \mathrm{H}_{5} \mathrm{~N}$ 络合形成活性中心; $\mathrm{AlHCl}_{2} \cdot \mathrm{Et}_{2} \mathrm{O}$ 过量 时则由于所用 $\mathrm{AlBr}_{3}$ 亦过量, 生成较多的 $\mathrm{AlBr}_{3} \cdot \mathrm{Et}_{2} \mathrm{O}$ 可能引起链终止的作用。

固定 $\mathrm{AlHCl}_{2} \cdot \mathrm{Et}_{2} \mathrm{O}$ 及 $\mathrm{AlBr}_{3}$ 用量, 改变 $\mathrm{CoCl}_{2}$. $4 \mathrm{C}_{5} \mathrm{H}_{5} \mathrm{~N}$ 用量 (以 $\mathrm{Co}$ 占单体重量表示) 则 3 小时转 化率随该用量增加而增加 (图 3); 分子量则降低。 Co 的用量少至单体用量的 $10 \mathrm{ppm}$ 时, 佮能聚合; 而在 $40 \mathrm{ppm}$ 以上时转化率有下降趋势，可能 $40 \mathrm{ppm}$ 是处于活性最大的 $\mathrm{Al} / \mathrm{Co}$ 比 (郎 $\mathrm{Al} / \mathrm{Co}$ 比=125)。 $\mathrm{CoCl}_{2} \cdot 4 \mathrm{C}_{5} \mathrm{H}_{5} \mathrm{~N}$ 量再增加, 由于 $\mathrm{AlHCl}_{2} \cdot \mathrm{Et}_{2} \mathrm{O}$ 用量

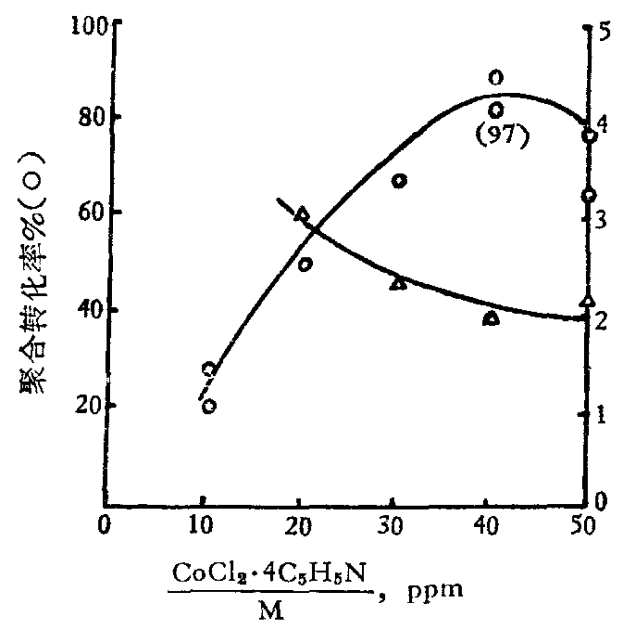

图 $3 \mathrm{CoCl}_{2} \cdot 4 \mathrm{C}_{5} \mathrm{H}_{5} \mathrm{~N}$ 的用量与聚合转化率及产 物分子量的关系 $\left(\mathrm{AlHCl}_{2} \cdot \mathrm{Et}_{2} \mathrm{O} / \mathrm{M}=1.47 \%\right.$ ， $\left.\mathrm{AlBr}_{3} / \mathrm{AlHCl}_{2} \cdot \mathrm{Et}_{2} \mathrm{O}=0.5,30^{\circ} \mathrm{C}\right)$
固定，则 $\mathrm{Al} / \mathrm{Co}$ 比下降，因之转化率也下降。

以上聚合溫度均为 $30^{\circ} \mathrm{C}$ 。图 4 表示聚合溫度 对转化率及分子量的影响。由图可见, 聚合温度由 $10^{\circ} \mathrm{C}$ 变化至 $40^{\circ} \mathrm{C}, 3$ 小时聚合转化率改变不大。可 能溫度高时聚合速度快些, 但溫度低时活性井不差。

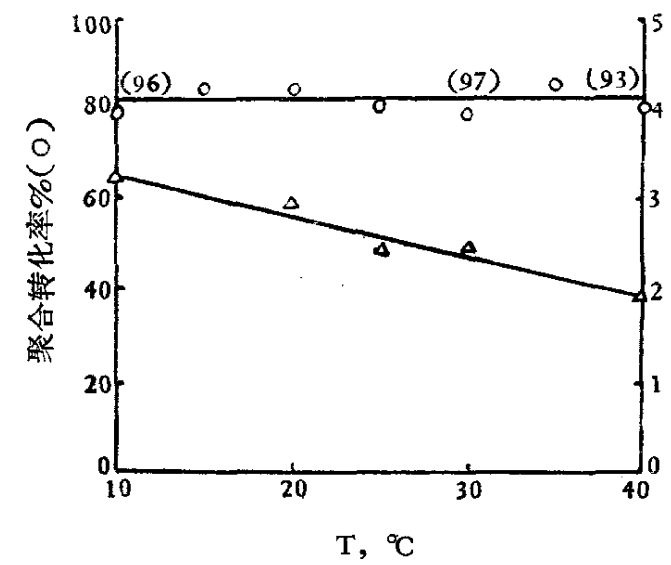

图 4 聚合溫度对聚合转化率及分子量的影响

$\left(\mathrm{AlHCl}_{2} \cdot \mathrm{Et}_{2} \mathrm{O} / \mathrm{CoCl}_{2} \cdot 4 \mathrm{C}_{3} \mathrm{H}_{5} \mathrm{~N}=100, \mathrm{Co} / \mathrm{M}\right.$ $=50 \mathrm{ppm}, \mathrm{AlBr}_{3} / \mathrm{AlHCl}_{2} \cdot \mathrm{Et}_{2} \mathrm{O}=0.5$ )

顺式 $-1,4$ 结构则溫度大于 $30^{\circ} \mathrm{C}$ 时有降低趋势, 这 与 $\mathrm{Et}_{2} \mathrm{AlCl}-\mathrm{CoCl}_{2} \cdot 4 \mathrm{C}_{5} \mathrm{H}_{5} \mathrm{~N}$ 的体系 ${ }^{[8]}$ 有类似处。分 子量则随聚合溫度增加而逐步下降。这说明溫度管 加, 链转移速度的增加大于链增长速度的增加。

系统中水分对聚合活性有很大的影响（图 5)。 以上聚合的水分均控制在 $25 \mathrm{ppm}$ 左右。增加水分则 转化率起初略有升高，水分再增大，则转化率逐步 下降。分子量随水分增大而增大，超过一定水分， 则产生㠜胶。这一结果与 $\mathrm{Et}_{2} \mathrm{AlCl}-\mathrm{CoCl}_{2} \cdot 4 \mathrm{C}_{5} \mathrm{H}_{5} \mathrm{~N}$ 的体系相仿 ${ }^{[8]}$ 。可能少量水与 $\mathrm{AlHCl}_{2} \cdot \mathrm{Et}_{2} \mathrm{O}$ 生成 的产物参与了活性中心的作用，井使活性中心键的

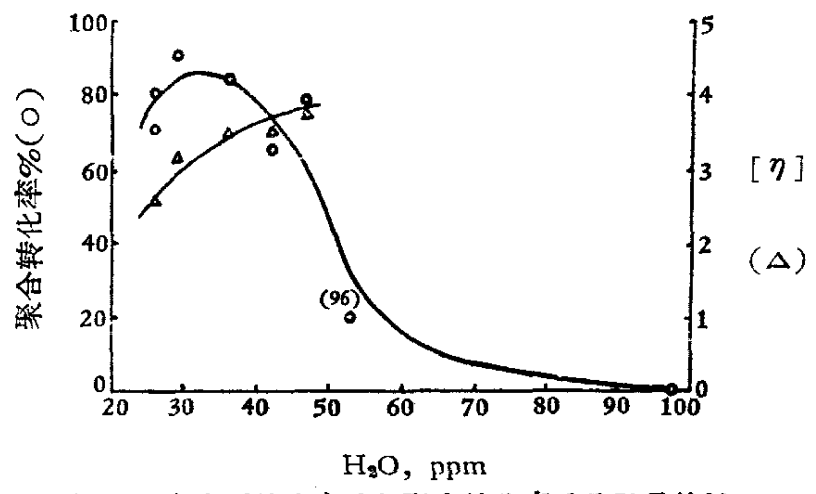

图 5 聚合系统中水分与聚合转化率及分子量的关 系 $\left(30^{\circ} \mathrm{C}, \mathrm{AlHCl}_{2} \cdot \mathrm{Et}_{2} \mathrm{O} / \mathrm{CoCl}_{2} \cdot 4 \mathrm{C}_{5} \mathrm{H}_{5} \mathrm{~N}=100\right.$, $\mathrm{Co} / \mathrm{M}=50 \mathrm{ppm}, \mathrm{AlBr}_{3} / \mathrm{AlHCl}_{2} \cdot \mathrm{Et}_{2} \mathrm{O}=0.5$ ) 
稳定性增加。多量水与 $\mathrm{AlHCl}_{2} \cdot \mathrm{Et}_{2} \mathrm{O}$ 作用产生的 副产物具有较强的阳离子催化剂的性质, 当聚合物 链增长至一定长度 后便交联 而产生凝胶。当水较 $\mathrm{AlHCl}_{2}$ 为过量时，则聚合被抑制。

以庚烷代替部分苯作为溶剂时转化率及分子量 均逐步下降（图 6)。顺式-1,4结构则改变不大。这可 能是由于大 $\pi$ 键化合物与活性中心键的稳定性及活 性有关。以不具有 $\pi$ 电子云的庚烷代替苯时, 则活性 中心键的稳定性及活性均減少。前者意味着链转移 容易发生, 导致分子量降低, 后者引起转化率下降。

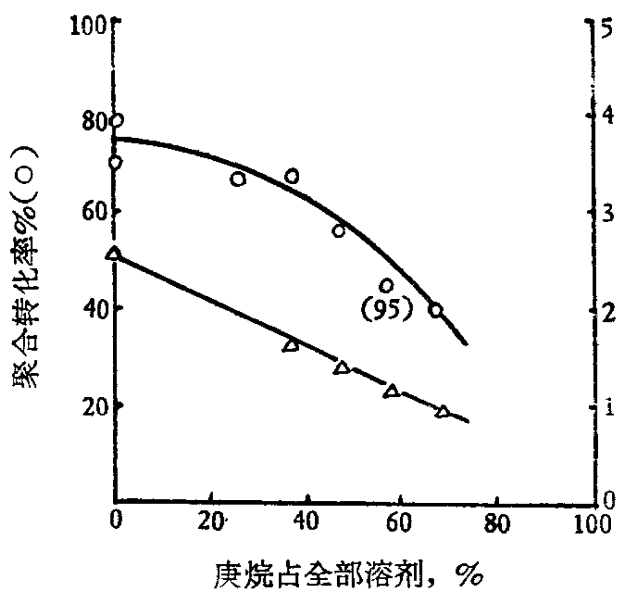

$[\eta]$

七

图 6 庚烷代替部分苯作为溶剂时对聚合转 化率及分子量的影响 (聚合条件同图 5)

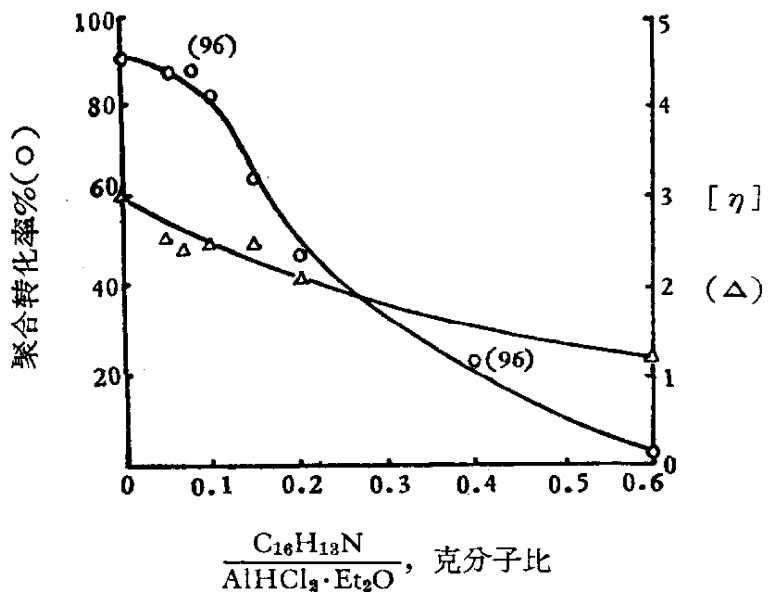

图 $7 \mathrm{~N}$-萊基- $\beta$-䒨胺对聚合的影响（除 $\mathrm{AlBr}_{3}$ / $\mathrm{AlHCl}_{2} \cdot \mathrm{Et}_{2} \mathrm{O}=0.6$ 外，聚合条件同图 5)

在易产生凝胶的条件下, 邲 $\mathrm{AlBr}_{3} / \mathrm{AlHCl}_{2} \cdot \mathrm{Et}_{2} \mathrm{O}$ 比等于 0.6 时，加入 $N$-苯基 $-\beta$-弥胺可抑制凝胶的 产生。当 $N$-苯基- $\beta$-萗胺 $/ \mathrm{AlHCl}_{2} \cdot \mathrm{Et}_{2} \mathrm{O}$ 的克分子 比小于 0.1 时，转化率下降很少 (图 7); 超过 0.1
时则转化率随 $N$-苯基- $\beta$-菜胺的增加而显著下降。 分子量则随 $N$-苯基- $\beta$-蔡胺的用量 增加而逐步下 降，至于产物的顺式 $-1,4$ 结构则几乎不受影响。 $N$-苯基- $\beta$-萗胺抑制凝胶的作用可 能是通过它与过 量的 $\mathrm{AlBr}_{3}$ 络合，使之不再起交联 聚合物链的作 用；但用量过多时则可能同时与活性中心中的鈷络 合，导致对聚合的抑制。

以上聚合时的加料方式均系在丁二烯溶液存在 下先加 $\mathrm{CoCl}_{2} \cdot 4 \mathrm{C}_{5} \mathrm{H}_{5} \mathrm{~N}$, 再加 $\mathrm{AlHAl}_{2} \cdot \mathrm{Et}_{2} \mathrm{O}$, 最后 加 $\mathrm{AlBr}_{3}$ 。如将加料方式改变, 则聚合活性有所不 同。其中以 $\mathrm{AlHCl}_{2} \cdot \mathrm{Et}_{2} \mathrm{O}$ 与 $\mathrm{AlBr}_{3}$ 先混合较好; $\mathrm{CoCl}_{2} \cdot 4 \mathrm{C}_{5} \mathrm{H}_{5} \mathrm{~N}$ 与 $\mathrm{AlBr}_{3}$ 先混合则活性较差。这或 许是由于 $\mathrm{AlBr}_{3}$ 会夺去 $\mathrm{CoCl}_{2} \cdot 4 \mathrm{C}_{5} \mathrm{H}_{5} \mathrm{~N}$ 上的吡啶, 使 $\mathrm{CoCl}_{2}$ 沉出，而使活性中心減少。无丁二烯存 在时, $\mathrm{CoCl}_{2} \cdot{ }_{4} \mathrm{C}_{5} \mathrm{H}_{5} \mathrm{~N}$ 与 $\mathrm{AlHCl}_{2} \cdot \mathrm{Et}_{2} \mathrm{O}$ 先反应, 则活性也不高。如在丁二烯溶液存在下先加 $\mathrm{AlBr}_{3}$, 则 $\mathrm{AlBr}_{3}$ 与丁二烯溶液中少量水分杂质作用，水解 而产生沉淀，使活性也降低。

本工作还试验了以 $\mathrm{AlHBr}_{2} \cdot \mathrm{Et}_{2} \mathrm{O}$ 代替 $\mathrm{AlHCl}_{2}$. $\mathrm{Et}_{2} \mathrm{O}$ 与 $\mathrm{CoCl}_{2} \cdot{ }_{4} \mathrm{C}_{5} \mathrm{H}_{5} \mathrm{~N}$ 及 $\mathrm{AlBr}_{3}$ 组成的三元催化 剂，发现也能使丁二烯进行顺式-1，4 聚合，活性 很高。以二乙酰基丙酮镍代替 $\mathrm{CoCl}_{2} \cdot 4 \mathrm{C}_{5} \mathrm{H}_{5} \mathrm{~N}$ 作 为上述 催化剂 组分，也能使丁二烯 聚合。活性 $\mathrm{Al} / \mathrm{Ni}$ 比在 100 至 250 左右。转化率及分子量均较 低，顺式 $-1 ， 4$ 结构也较低，仅 $91 \%$ 左右。

誌谢：本交承吴学周所长于百记中审阅，特此致谢。

[1] Mazzei A., Araldi M., Marconi W., De Malde M., Polymer Letters, 1, 79 (1963).

[2] Marconi W., Mazzei A., Araldi M., De Malde M., Chim. Ind. 46, 245 (1964).

[3] 谢洪泉、李平生, 中国科学院应用化学研 究 所 集 刊，第 6 集, 60(1962)。

[4] Wiberg E., Schmidt M., Z. Naturforschg., 6b, 459, 460 (1951).

[5] Nicholson D. G., Winter P. K., Fineberg H., Inorganic Synthesis, III, 33 (New York-London, 1950).

[6] Katzin L. I., Ferraro J. R., Gebert E.,J. Am. Chem. Soc., 72, 5471 (1950).

[7]谢洪泉、单新忠, 高分子通讯, 7, 18(1965)。

[8] Gippin M., Ind. Eng. Chem., Product Research and Development, 1, 32 (1962); 唐学明、杨超 雄、赵普康，高分子通讯，5，49(1963)。 